\title{
Ultrasonographic Findings of Uterine Artery Pseudoaneurysm
}

\author{
Sayuri Kondo ${ }^{a}$, Shunji Suzukia, b
}

\begin{abstract}
A 30-year-old woman, gravid 1, para 1 underwent an emergency cesarean section (CS) at 40 weeks of gestation because of intrauterine infection. Seven hours after CS, sudden massive vaginal bleeding of $1,500 \mathrm{~mL}$ occurred. Transvaginal ultrasonography with color Doppler revealed the presence of low density cyst with a diameter of $4 \mathrm{~cm}$ with spiral pulsating blood flow in the cavity of the cyst in the vicinity of the ascending branches of the left uterine artery. We diagnosed it as uterine artery pseudoaneurysm, and total hysterectomy was performed because of unstable vital signs under transfusion.
\end{abstract}

Keywords: Uterine artery pseudoaneurysm; Cesarean section; Transvaginal ultrasonography

\section{Introduction}

Pseudoaneurysm is a dilation of an artery with partial or full disruption of the vessel wall [1-4]. Uterine artery pseudoaneurysm (UAP) is rare but a potentially life-threatening lesion. Although many clinical interventions have been postulated to be responsible for development of UAP, cesarean section (CS) has seemed to be the most frequent cause of UAP because CS has possibilities of causing vessel injuries involving branches of uterine arteries [3]. The initial usefulness of ultrasonography for suspecting UAP has been well documented [1, 2, 4]. The unexpected appearance of uterine anechoic lesions at ultrasonography can suggest the presence of UAP. The utilization of color Doppler seems to assist the confirmed diagnosis of UAP. Within the UAP, in addition, swirling arterial flow with different directions and velocities has been reported to be observed like the current case [2]. We present here a case of UAP following emergency CS diagnosed by ultrasonography.

Manuscript submitted June 26, 2017, accepted July 7, 2017

aDepartment of Obstetrics and Gynecology, Japanese Red Cross Katsushika Maternity Hospital, Tokyo, Japan

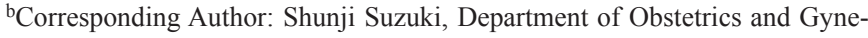
cology, Japanese Red Cross Katsushika Maternity Hospital, 5-11-12 Tateishi, Katsushika-ku, Tokyo 124-0012, Japan. Email: czg83542@mopera.ne.jp

doi: https://doi.org/10.14740/jmc2867w

\section{Case Report}

A 30-year-old woman, gravid 1, para 1 with no previous disease or family history underwent an emergency CS at 40 weeks of gestation because of intrauterine infection and fetal asphyxia. A male infant weighing 2,922 $\mathrm{g}$ was born with Apgar scores of 8 and 9 at 1 and $5 \mathrm{~min}$, respectively. Histopathology of the placenta did not demonstrate any abnormal findings except acute funisitis with chorioamnionitis. Total blood loss during surgery was $500 \mathrm{~mL}$. The clinical course until several hours after $\mathrm{CS}$ was uneventful without any further complications. Seven hours after CS, sudden massive vaginal bleeding of $1,500 \mathrm{~mL}$ occurred. The hemoglobin concentration decreased to $6.6 \mathrm{~g} /$ $\mathrm{dL}$. And then, genital bleeding of about $1,000 \mathrm{~mL} / \mathrm{h}$ persisted for $2 \mathrm{~h}$. Transvaginal ultrasonography with color Doppler revealed the presence of low density cyst with a diameter of 4 $\mathrm{cm}$ with spiral pulsating blood flow in the cavity of the cyst in the vicinity of the ascending branches of the left uterine artery (Fig. 1). The arterial blood flows like a jet into the cavity during systole were also observed (Fig. 2).

We diagnosed it as UAP, and total hysterectomy was performed because of unstable vital signs under transfusion.

\section{Discussion}

In the current case, we were able to diagnose UAP only by ultrasonography; however, the diagnosed period seemed to be too late unfortunately. Gray-scale ultrasound findings show a well-defined complex echogenic mass different in appearance from the rest of the placenta. Prompt and accurate diagnosis should be made using color Doppler ultrasonography to avert life-threatening bleeding since it plays a significant role in demonstrating the vascular nature of this anechoic uterine lesion. If the earlier diagnosis of smaller UAP could be performed, an informed consent concerning arterial embolization might be possible to be performed. Based on the current case, we realized again the importance of prompt ultrasonographic examination in cases of massive postpartum hemorrhage.

\section{Conflicts of Interest}

The authors declare that there are no conflicts of interest regarding the publication of this paper. 


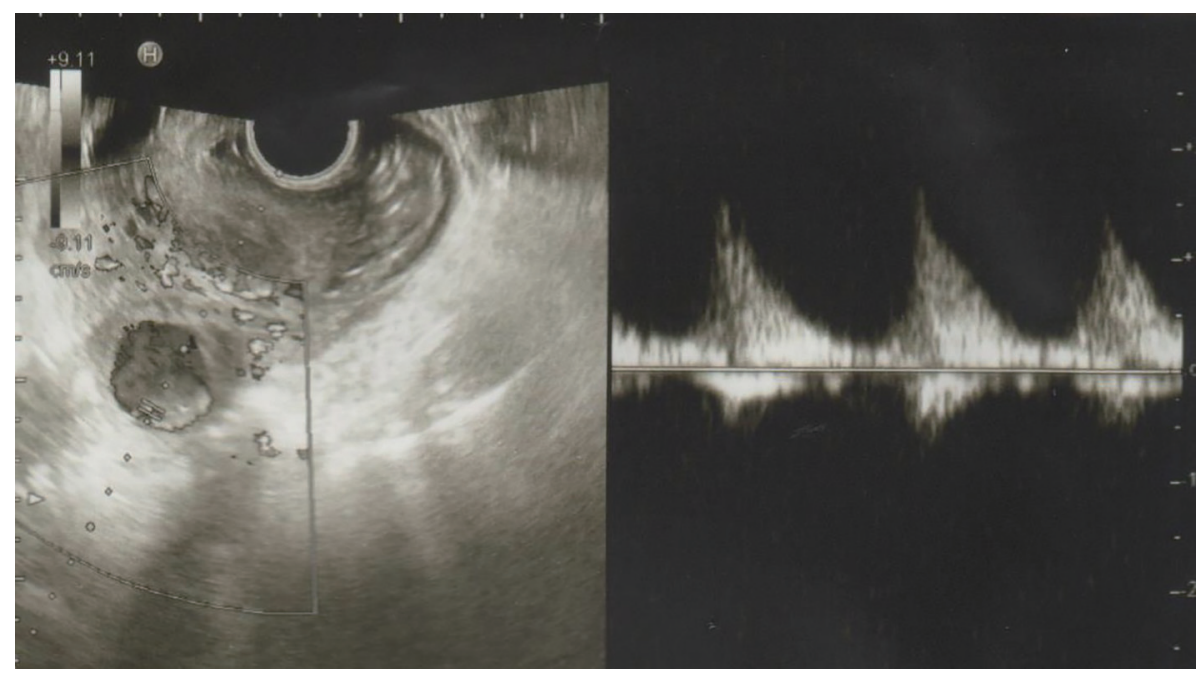

Figure 1. Transvaginal ultrasonography showing the presence of low density cyst with a diameter of $4 \mathrm{~cm}$ with spiral pulsating blood flow in the cavity of the cyst in the vicinity of the ascending branches of the left uterine artery.

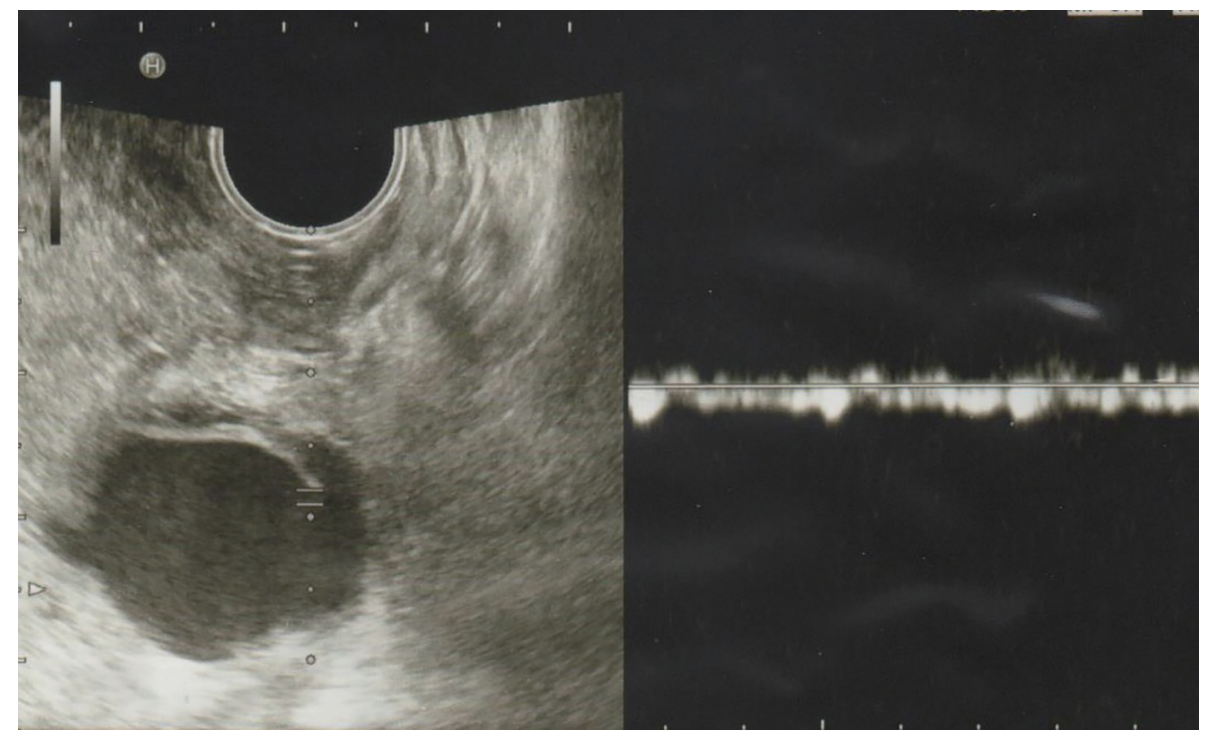

Figure 2. Transvaginal ultrasonography showing the arterial blood flows like a jet into the cavity during systole.

\section{References}

1. Ciebiera M, Slabuszewska-Jozwiak A, Zareba K, Jakiel G. Management of uterine artery pseudoaneurysm: advanced ultrasonography imaging and laparoscopic surgery as an alternative method to angio-computed tomography and transarterial embolization. Wideochir Inne Tech Maloinwazyjne. 2017;12(1):106-109.

2. Karmous N, Ayachi A, Derouich S, Mkaouar L, Mourali
M. Rupture of uterine artery pseudoaneurysm: role of ultrasonography in postpartum hemorrhage management. Pan Afr Med J. 2016;25:136.

3. Lausman AY, Ellis CA, Beecroft JR, Simons M, Shapiro $\mathrm{JL}$. A rare etiology of delayed postpartum hemorrhage. J Obstet Gynaecol Can. 2008;30(3):239-243.

4. Zimon AE, Hwang JK, Principe DL, Bahado-Singh RO. Pseudoaneurysm of the uterine artery. Obstet Gynecol. 1999;94(5 Pt 2):827-830. 